\title{
NARROWBAND ULTRASONIC SPECTROSCOPY FOR NDE OF LAYERED STRUCTURES
}

T. Stepinski and M. Jonsson

${ }^{1}$ Uppsala University, Uppsala, Sweden

\begin{abstract}
NDE of airspace sandwich structures is often performed using a simple resonant transducer sensing the information in the frequency domain obtained due to the constructive and destructive interference of elastic waves. Application field of ultrasonic narrowband ultrasonic spectroscopy (NBUS) is likely to increase rapidly with the growing application of layered structures in modern aircraft, for example, GLARE. The aim of this paper is to enlighten the potential and the limitations of the NBUS techniques. Operation of the NBUS technique based on the measurements performed using a specially designed resonance transducer with carefully selected narrow frequency band is explained in detail. Novel method based on the use of impedance plane for sensing transducer resonance is presented. Theoretical results illustrate the relation of the impedance plane indications to the adhesive strengths in aluminum sandwich structures. Finally, practical application of the proposed technique on mechanized inspection of carbon fiber reinforced structures is presented.
\end{abstract}

Introduction: Ultrasonic resonance spectroscopy (URS) utilizes information in the frequency domain obtained due to the constructive and destructive interference of elastic waves for nondestructive evaluation of inspected objects. In a resonance test an ultrasonic tone-burst with sweeping frequency is applied to an ultrasonic transducer and a resonance spectrum of the inspected structure is acquired, [1]. The acquired spectrum contains information about the properties of materials used for the structure and flaws that may be present in it. Generally, there are two types of resonance test, global and local. Global tests provide synthetic information about the entire inspected part, and therefore, can be applied to relatively small parts where vibrations can be excited in the whole inspected volume. Local tests are more suitable for aerospace structures, where local structure condition is of interest, and only a selected part of the structure is excited.

Ultrasonic resonance spectroscopy has been used for the inspection of aerospace structures and its application field is expected to increase rapidly with the growing application of layered structures in modern aircraft (e.g. GLARE that is a structure material used for the A380 from Airbus). However, the new applications compel increased demands on the RI instruments and transducers in terms of their sensitivity and their ability to characterize defects. Unfortunately, very limited information concerning the operation principles of the commercially available RI instruments is available in the literature.

A fundamental limitation of resonance inspection is its sensitivity to factors that are unessential for the test, such as, variations in dimensions or material properties that may mask the effect of smaller defects. This problem can be solved either by modeling or, when modeling is unfeasible by employing sophisticated self-learning algorithms for tuning parameters of the defect detector. In the case of multilayered aerospace structures modeling seems to be the most suitable solution. The purpose of the present paper is to fill this gap and to enlighten the potential and the limitations of the URS techniques. The presented material is a result of our research aimed at development of narrowband ultrasonic spectroscopy (NBUS) capable of coping with new applications and the increased demands. We start from explaining NBUS principles for multilayered structures and presenting a model enabling simulation of the tested structure and transducer. Finally, we present a new solution to the sensor problem based on measuring its electrical impedance. 
Measurement setup: Narrowband ultrasonic spectroscopy has been used for the NDE of aerospace multi-layered structures since 70-ties. Commercially available instruments (bond testers) used for this test operate on the principle of exciting a mechanical resonance in a multilayered structure. A piezoelectric probe, excited by a swept frequency signal is coupled to the surface of the inspected structure using a coupling agent (see Fig. 1). The instrument acquires probe's frequency spectrum in the range of some tens of $\mathrm{kHz}$ to several hundreds of $\mathrm{kHz}$. A resonance in the layered structure occurs when echoes between two boundaries travel back and forth due to the difference in acoustic impedances at the boundaries. For multi-layered structures, a number of resonance modes can be observed depending on their geometry and condition. A characteristic resonance pattern, an ultrasonic signature, obtained for each particular defect-free structure and given transducer can be used as a reference.

It is worth to mention that the probe characteristics are crucial for the NBUS test since shift of its resonance frequency indicates condition of the inspected structure. Therefore highly resonant piezoelectric probes are preferred for this kind of test. Serious difficulties can be encountered when applying NBUS to attenuating materials, like carbon fiber reinforced composites (CFRP). Resonance of a probe coupled to such structure is not only shifted in frequency but also spoiled in the sense that the resonance peak becomes broad and the resonance frequency cannot be detected accurately.

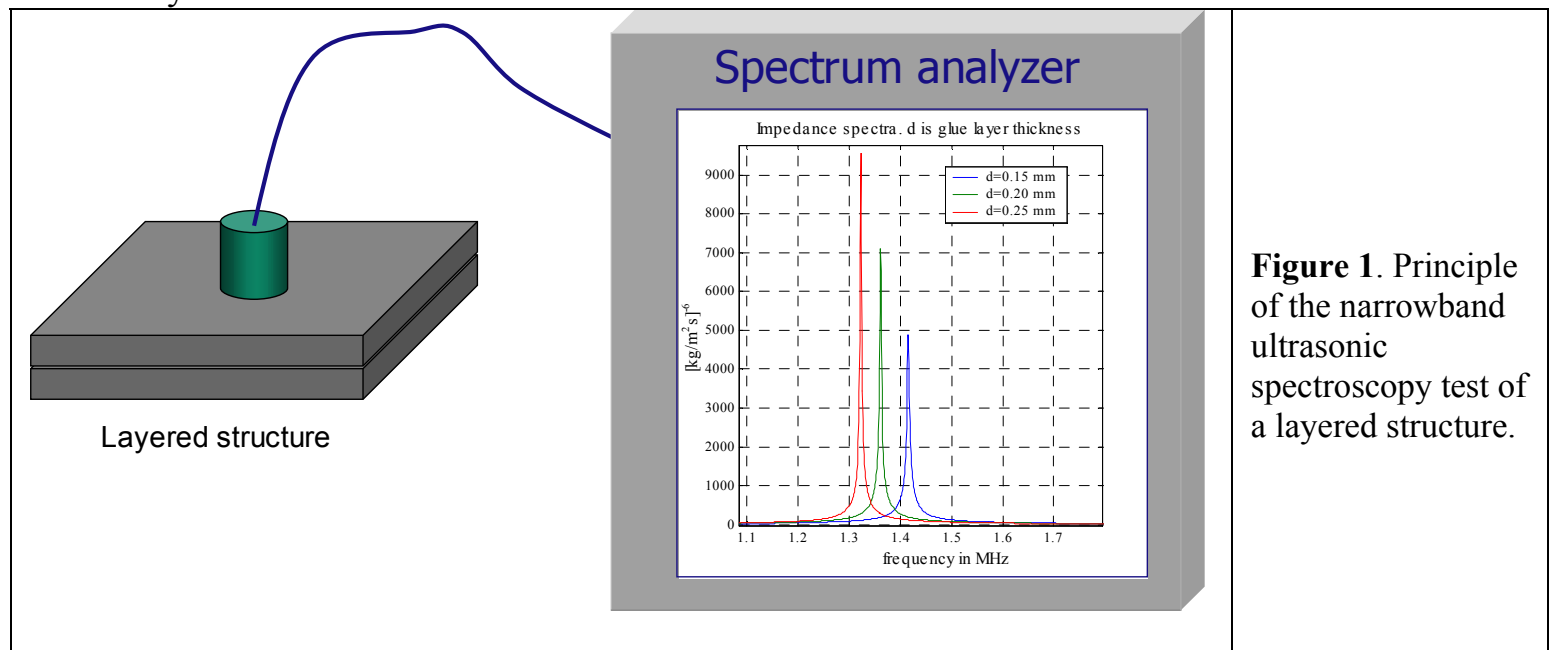

In classical NBUS inspection of an unknown object (for instance, using Fokker Bond Tester) its surface is scanned using a suitable probe and the resulting ultrasonic spectrums are acquired for a number of frequencies. Debond detection is performed by an operator observing simple features of the acquired spectra, such as, center frequency and amplitude of the highest peak in a preselected frequency range. This means that the operator has to perform the spectrum classification task based on primitive features extracted by the instrument. Below, we present models that explain this principle.

Structure modeling: A software tool was developed for calculating the input acoustical impedance of sandwich structures consisting of a number of semi-infinite parallel layers. Material constants, such as density and sound velocity are used as parameters. The software, which is written in Matlab ${ }^{\mathrm{TM}}$ uses a model based on the transmission line concept for recursive calculation of the impedances seen from the top of the successive layers. It is assumed that a longitudinal plane wave is incident on the structure's top boundary, details can be found in [2], [6] and [7]. Material attenuation can be included in the model. 
Assuming that acoustical impedances and thicknesses of the individual layers are known the model implemented in the toolbox calculates the impedance of the multi-layered structure. The model is valid for longitudinal plane waves only.

The software has been used for simulating laminated airspace structures consisting of aluminum layers bonded by adhesive layers. Spectral variations due to debonds and voids between the aluminum plate and the adhesive can be modeled and evaluated. As an example, Fig. 2 shows results obtained for a simple laminated structure consisting of two aluminum plates (top $1.27 \mathrm{~mm}$ and bottom $1.0 \mathrm{~mm}$ ) bonded with $0.2 \mathrm{~mm}$ adhesive.

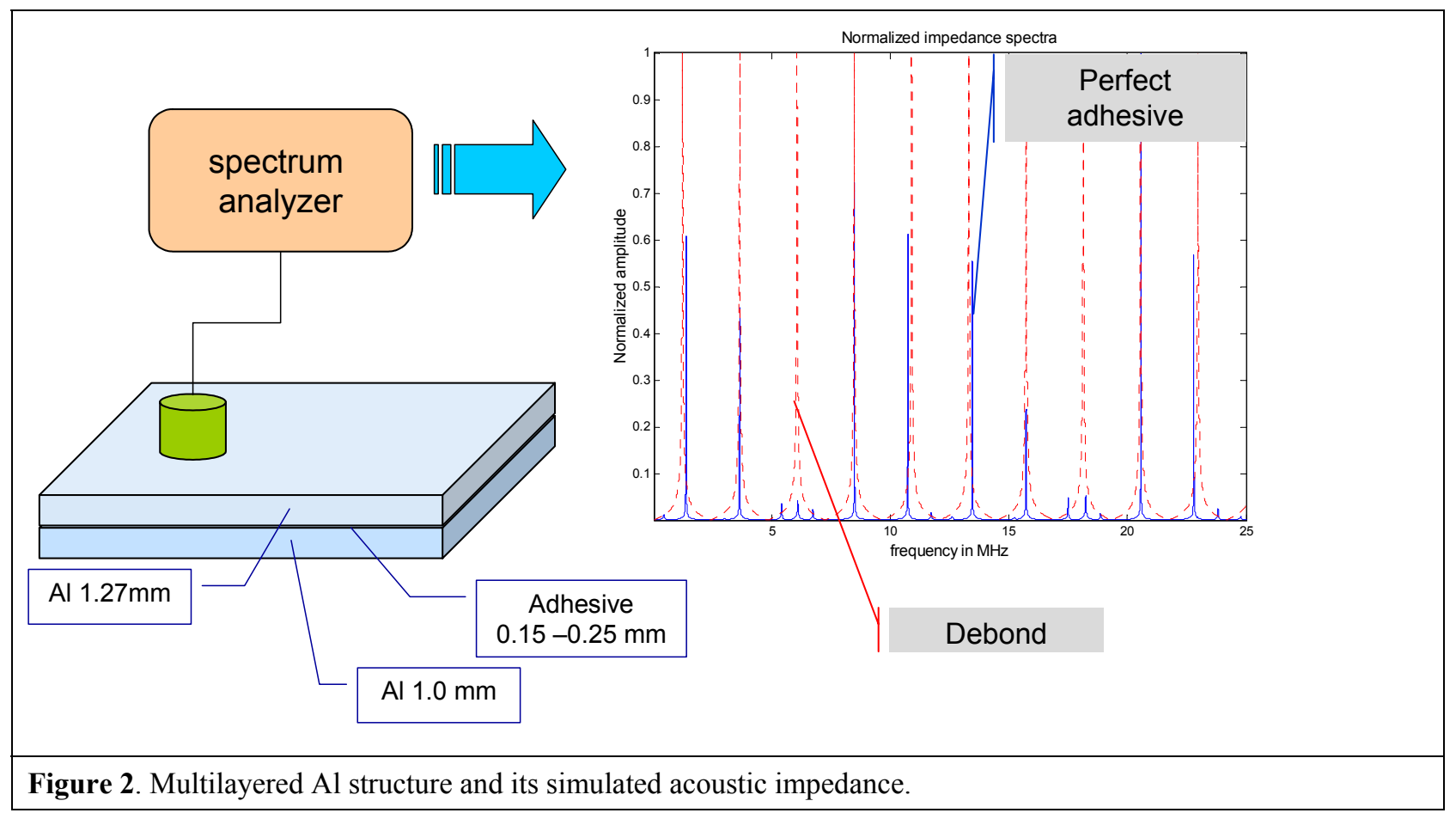

Debonds would be relatively easy to detect if variations in the spectra measured for a defect free structure (nominal spectra) were small. Unfortunately, variations of the adhesive parameters (mainly its thickness) may result in quite large shifts of the resonance peaks.

Transducer modeling: A narrowband, piezoelectric low frequency transducer (below $1 \mathrm{MHz}$ ) is a vital part of the NBUS setup. Since the transducer is undamped, it has a distinct frequency peak corresponding to its thickness mode (cf. [3]).

Piezoelectric transducers are electromechanical systems that can be modeled in many ways depending on the application. Here, the KLM model [4], based on the transmission line concept was chosen since it enables modeling the transducer under various load conditions. In the KLM model the acoustical side (a transmission line of a length $d$ ) is coupled to the electrical side by a transformer with ratio (1: $\phi$ ) (see Fig. 3). The transmission line has two ports where acoustical loads are applied, the backing at the left-hand side and the inspected structure at the right-hand side. Thus, the influence of acoustical load variations on the transducer's electrical impedance can be modeled.

In other words, when a piezoelectric transducer is used for inspecting a multi-layered structure, as shown in Fig. 3, the structure's acoustical impedance can be directly plugged 
into the KLM model and the electrical impedance of the transducer calculated using this model will reflect variations in the inspected structure.

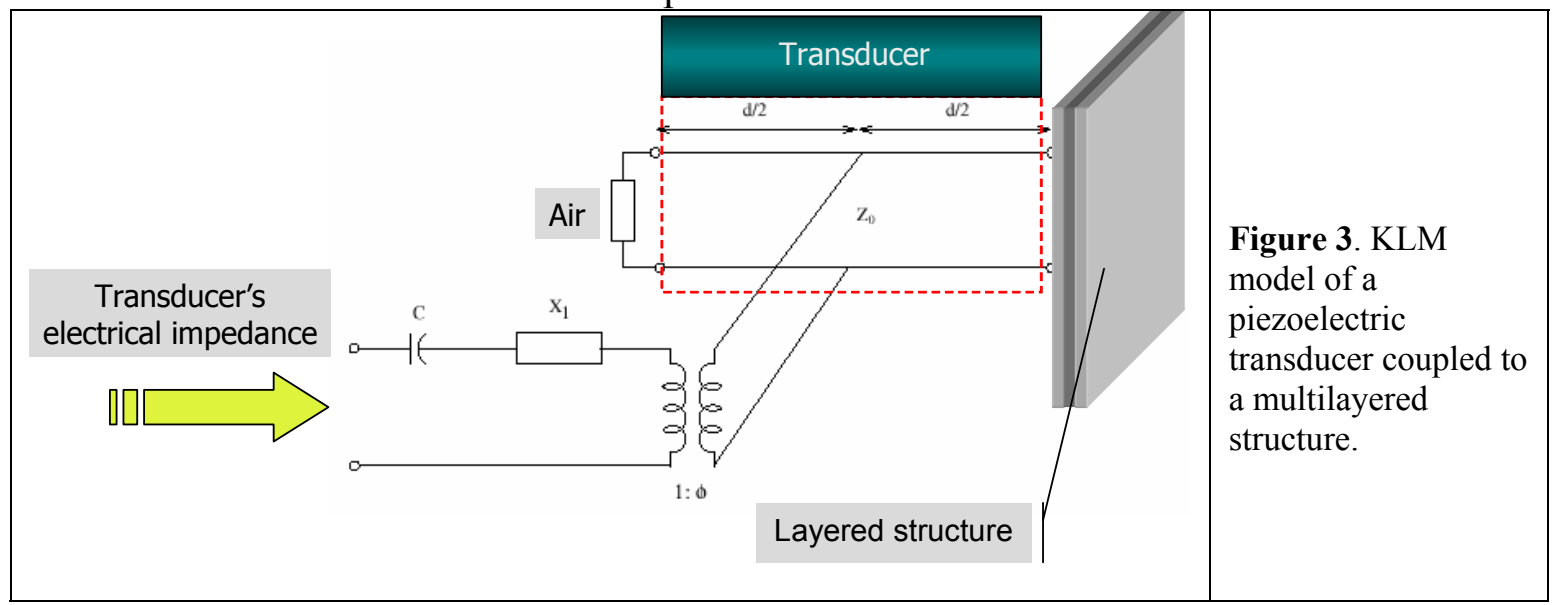

Before this can be done parameters of the transducer model have to be estimated using an adequate procedure. Fig. 4 shows as an example results obtained for a simple piezoelectric transducer (PZT cylinder with diameter approx. $13 \mathrm{~mm}$ and thickness $25 \mathrm{~mm}$ ). The electrical admittance of this element, measured using the 4395 A Network/ Spectrum/Impedance Analyzer from Agilent Technologies is shown in Fig. 4 together with the results obtained from the KLM model. Parameters of the KLM model corresponding to the transducer were estimated from the Analyzer measurements using an identification procedure described in [6]. A very good agreement between the measurements and simulations was obtained, which is not surprising for an undamped transducer in air.

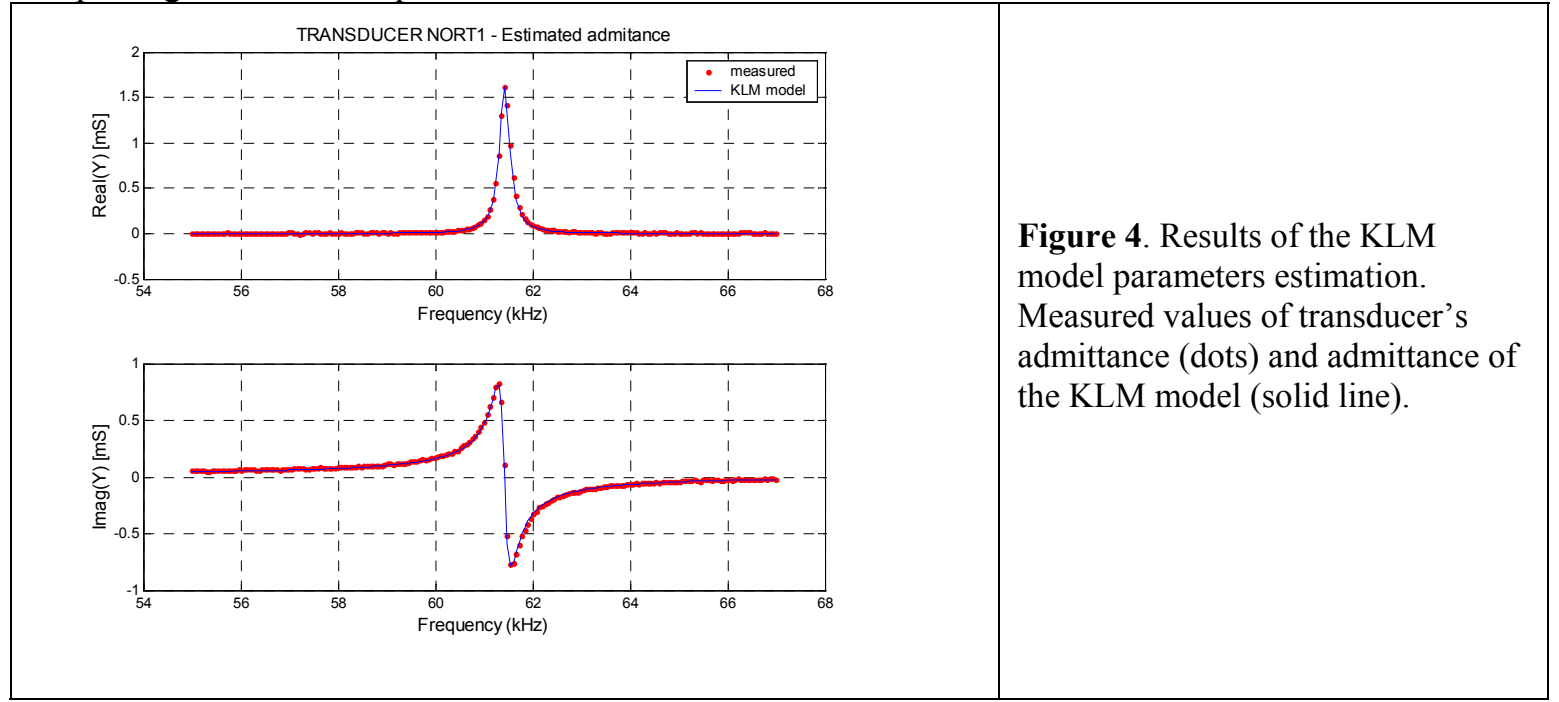

When the transducer is loaded with an acoustic load (e.g. it is coupled to a multi-layered structure) its spectrum changes depending on the acoustic impedance of the load and the resonance frequency shifts slightly. These changes can be detected if the transducer is excited with a swept frequency signal and its electrical impedance measured in some frequency band is displayed for an operator.

An attempt was made to use an electronic circuit employing a phase locked loop (PLL) circuit for detecting resonance frequency of a piezoelectric resonator. The automatic detector was tested on triple-layered Al structures with artificial debonds, the results were presented in [6] and [7]. The measurements and the simulations have shown a significant difference between the resonance frequencies corresponding to lower and upper debonds in Al structures. The above-presented 
software tool enabled maximizing this difference appropriately to the type of structure by the choice of frequency band optimal for the applied transducer. However, sensitivity of this method was sufficient neither for the evaluation of weak bonds produced in $\mathrm{Al}$ samples nor for detecting defects in CFRP.

Poor results obtained in the demanding applications motivated the development of a new method based on sensing transducer's complex valued impedance for a single, carefully selected frequency.

Variations of the transducer impedance: Transducer impedance is a complex number that can be represented as a vector in a complex plane. Modeling results have shown that transducer impedance measured for some frequency close to its resonance underlies considerable variations depending on condition of the inspected material.

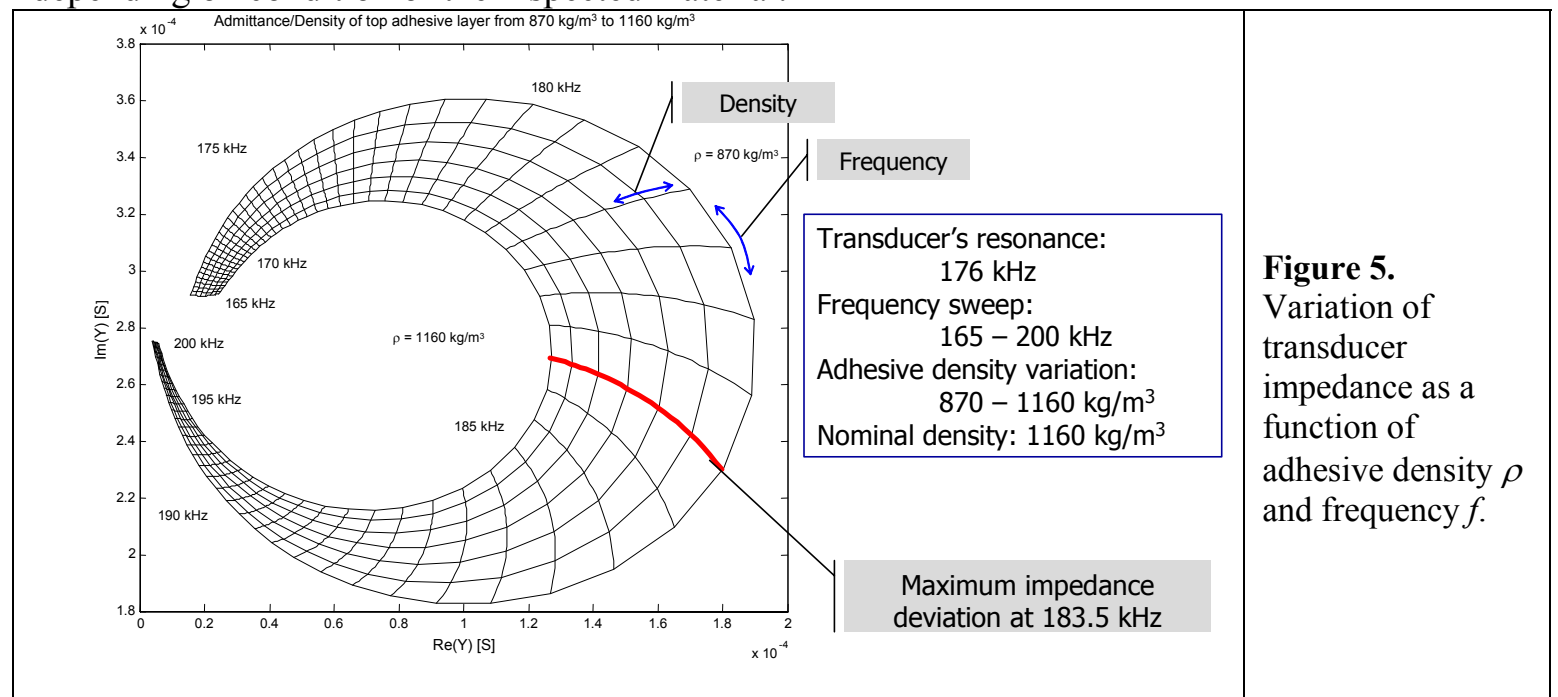

When the inspected surface is scanned using a piezoelectric probe coupled to the inspected structure the impedance vector in the impedance plane moves providing additional information, like in eddy current inspection.

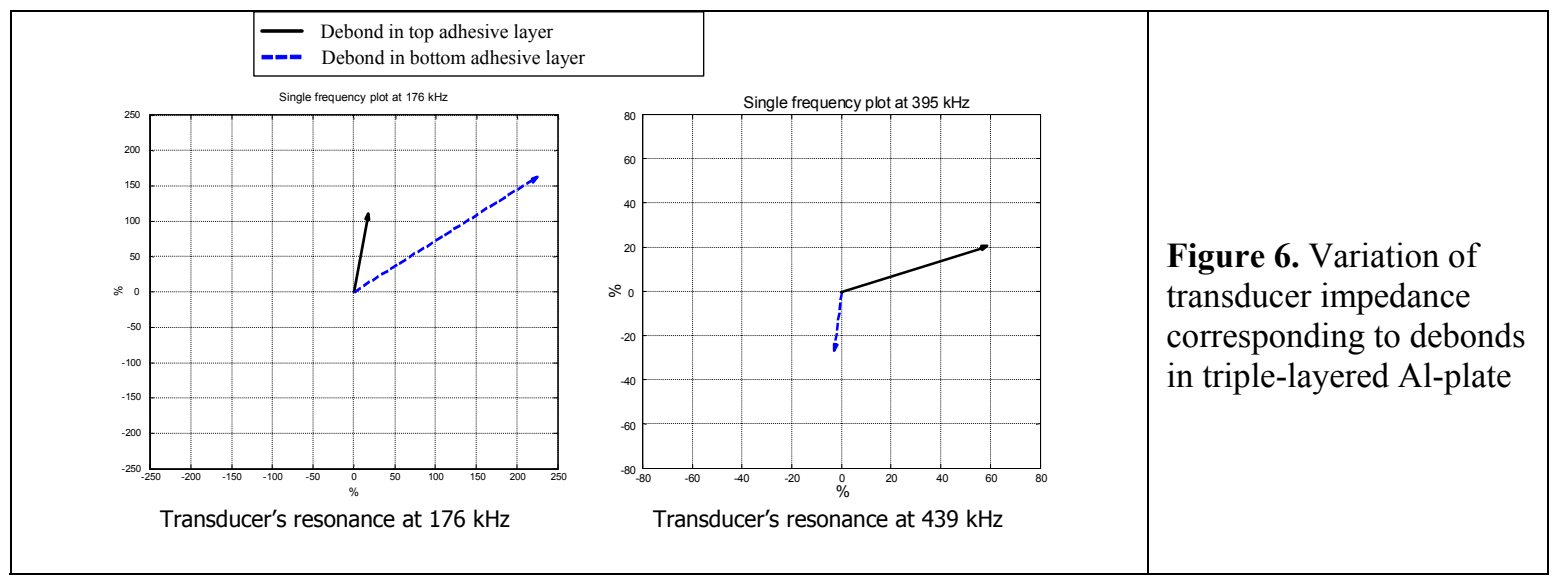

An example of impedance variation for the Fokker probe used in the above-mentioned experiment (cf. Fig. 2) in function of adhesive mass density $\rho$ for the test frequencies in the interval from 165 to $200 \mathrm{kHz}$ is shown in Fig. 5. Frequency change moves the operating point along an arc while the increase of mass density moves it towards the coordinate origin. An example of impedance analysis is presented in Fig. 6 for a triple-layered Al structure with two adhesive layers, upper and lower. Transducer responses to debonds in the adhesive layers are 
shown for two operating frequencies. It is apparent that both debonds can be distinguished based on their responses in the impedance plane, however, at higher frequency $(395 \mathrm{kHz})$ an angle between the indications is larger (at the price of a reduced magnitude). Similarity to the variation of coil impedance in eddy current testing is apparent.

It should be noted that the Bond Master from Staveley NDT Technologies probably implements the principle similar to that presented here. Our modeling tool makes possible the systematic investigation of how different structure parameters (such as, debonds, adhesive strength) effect transducer impedance and in this way enables an optimal choice of a suitable probe and its test frequency with respect to the inspected structure. Below, we present some experimental results obtained in CFRP inspection.

Inspection of CFRP specimens: Mechanized inspection of CFRP samples was performed using an XY-scanner, computerized impedance measuring instrument and a PZT transducer operating at frequencies in the range of $1 \mathrm{MHz}$. The transducer had diameter 0.5 " and was fed with a single frequency sine wave with

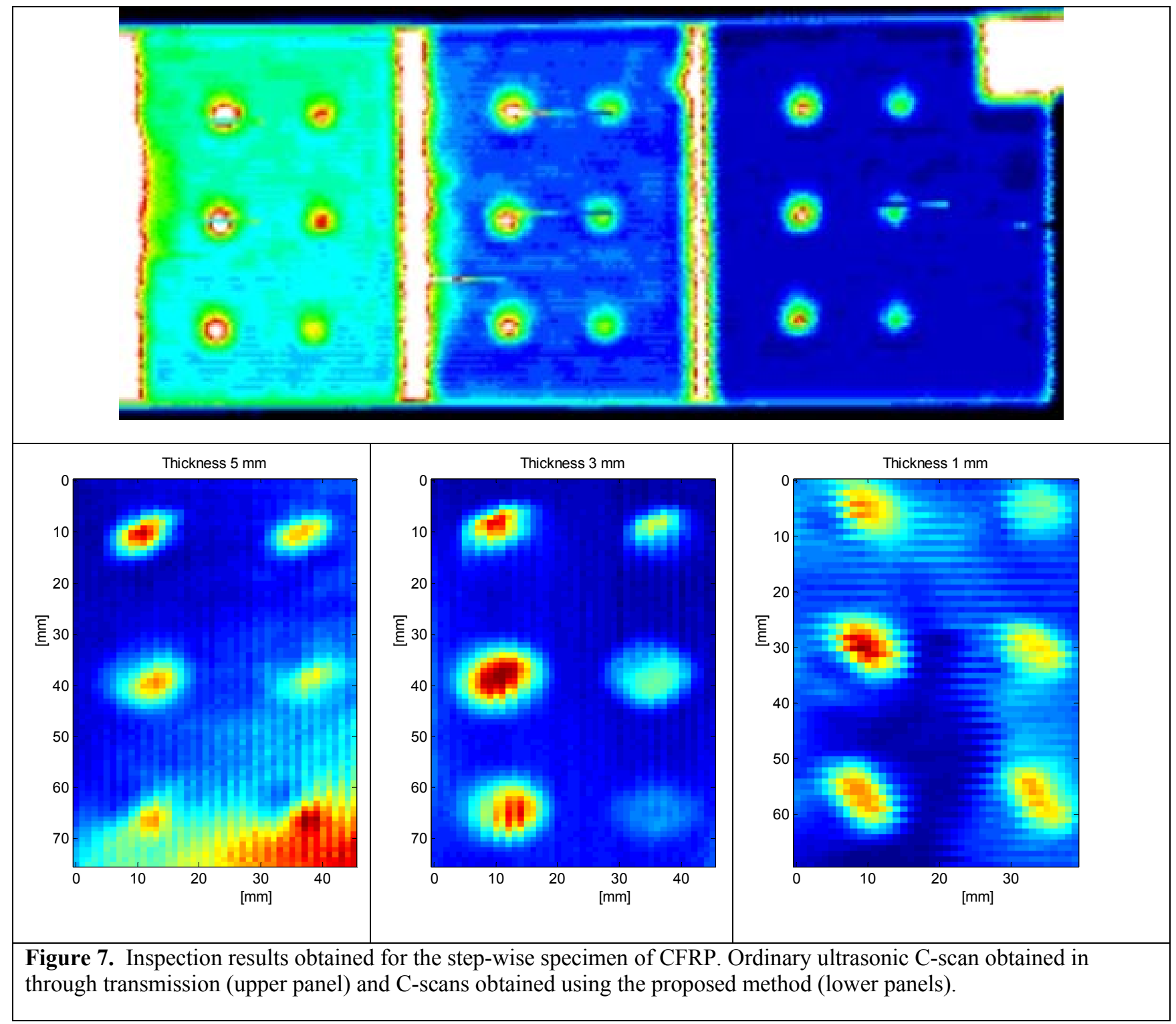


amplitude approx. $10 \mathrm{~V}_{\mathrm{p} \text {-p }}$ and its complex impedance measured in discrete $\mathrm{XY}$-points was stored in a PC.

A complex valued C-scan obtained in this way was displayed directly at the PC's screen. A small volume of water applied on the inspected surface was used to obtain contact between the transducer and the inspected structure. A step-wise specimen with variable step thicknesses $(1,3$ and $5 \mathrm{~mm}$ ) was inspected. In each step 6 artificial defects were manufactured to simulate delaminations, see Fig. 7. The defects manufactured at each step have two sizes, 6 x $6 \mathrm{~mm}$ (left column) and $4 \times 4 \mathrm{~mm}$ (right column in Fig. 7). The defects in each column were located at different depths, in the lower row in Fig. 6 are the defects located at the depth of two plies from the upper surface, in the upper low are the defects located at two plies from the lower surface, and the middle row represents defects in the middle of step thickness.

The results are shown in Fig. 7 together with the ultrasonic C-scan obtained in transmission inspection as a reference. It is evident that the proposed method is capable of detecting almost all defects in the inspected specimen; the most difficult seems to be detecting shallow defects in the thick section of $5 \mathrm{~mm}$. It should be noted however, that the presented results are preliminary and that the probe used in the experiments has not been optimized for this purpose. Nor have any attempts yet been made to improve SNR by averaging or other signal processing methods.

\begin{tabular}{|c|c|c|}
\hline Transducer's frequency $1 \mathrm{MHz}$ & Transducer's frequency $2 \mathrm{MHz}$ & \\
\hline 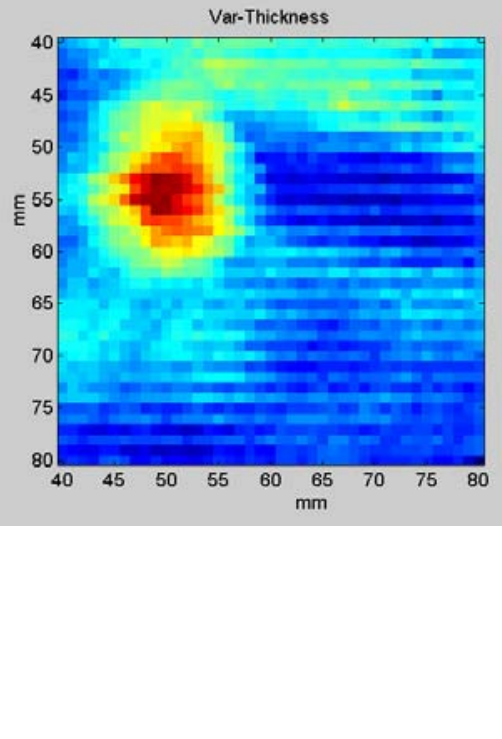 & 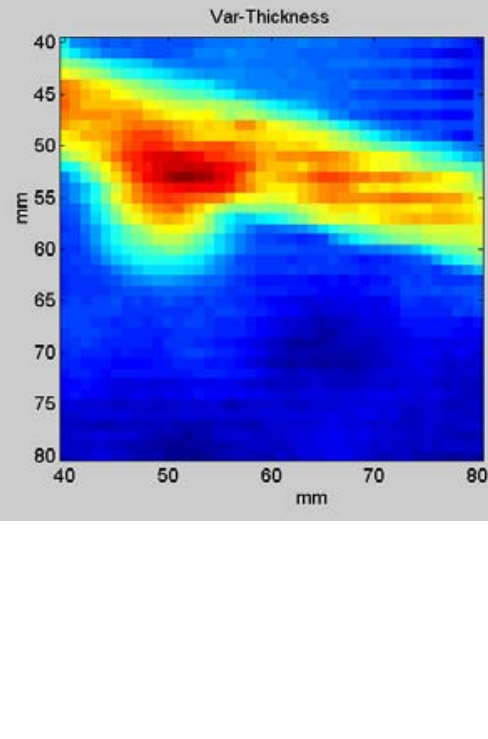 & $\begin{array}{l}\text { Figure 8. } \\
\text { Inspection } \\
\text { results } \\
\text { obtained for } \\
\text { a CFRP } \\
\text { specimen } \\
\text { with impact } \\
\text { and local } \\
\text { variation in } \\
\text { thickness at } \\
\text { two } \\
\text { frequencies, } \\
1 \mathrm{MHz}(\mathrm{left}) \\
\text { and } 2 \mathrm{MHz} \\
\text { (right). }\end{array}$ \\
\hline
\end{tabular}

The effect of test frequency is illustrated by an example shown in Fig. 8 presenting results of the inspection of a CFRP specimen with an impact. The inspection was performed using a broadband transducer operating at two frequencies, $1 \mathrm{MHz}$ and $2 \mathrm{MHz}$, respectively. Both C-scans in Fig. 8 correspond to the same area of the specimen. An impact in the specimen is well pronounced at the upper-left corner of the $\mathrm{C}$-scans at both frequencies. However, the inspected specimen had local variation in thickness taking the form of a line with increased thickness. This line can be easily seen at the right hand $\mathrm{C}$-scan acquired at $2 \mathrm{MHz}$.

Summary and conclusions: Feasibility of different URS set-ups in application to aerospace structures has been investigated and the narrowband spectroscopy (NBS) has been selected as the 
most promising candidate. The method based on tracing resonance frequency of a resonant transducer using a PLL circuit was developed and examined. It appeared that its sensitivity in more demanding applications was unsatisfactory. An alternative method based on sensing probe's electrical impedance was developed and verified using CFRP specimens with artificial and natural defects.

NBS tests of layered structures performed using a narrowband transducer require proper choice of transducer and its resonance frequency. Today, this choice is performed ad hoc, based on operator's experience and some recommendations from the instrument manufacturer. The theoretical model of the multi-layered structure including the piezoelectric transducer presented in the paper makes this choice much easier and enables maximization of the transducer indication with respect to the parameters of the inspected structure. The model enables accounting for the effects of natural variations in dimensions and material properties as well as optimizing sensitivity of the NBS transducer to defects. Simulation results obtained using the presented model were used to explain the principle of narrowband inspection of multilayered structures. The simulation results were verified by measurements performed using a network analyzer.

Preliminary mechanized tests performed on selected specimens yielded very promising results; the proposed method was capable of detecting artificial delaminations in CFRP of different thicknesses as well as natural impact defects.

Acknowledgments: This work was supported by two projects funded by the European Community: INCA (Contract No: G4RD-CT2001-00507) and NANOSCAN (Contract No: G4ST-CT2002-50288). I would like to thank my student Mr. R. Risberg who performed measurements published in this paper.

\section{References:}

1. A. Migliori and J.L. Sarrao. Resonant Ultrasound Spectroscopy, John Wiley \& Sons, Inc., 1997.

2. L.M. Brekhovskikh, Waves in Layered Media, Academic Press, 1980.

3. C.C.H. Guyott, P. Cawley and R.D. Adams, "Vibration characteristics of the Mk II Fokker Bond Tester probe", Ultrasonics, vol. 24, pp. 318-324,1986.

4. R. Krimholtz, D.A. Leedom and G.L. Matthaei, "New Equivalent Circuits for Elementary Piezoelectric Transducers", Electronic Letters, vol. 6, pp. 398-399, 1970.

5. T. Stepinski, "Ultrasonic spectroscopy of adhesively bonded muli-layered structures", Proc. of the $3^{\text {rd }}$ International Conf. Emerging Technologies in Non-Destructive Testing, pp. 89-94, 2003.

6. R. Risberg, "Modeling Multi-layered Structures for Resonant Inspection", M.Sc. Thesis, Uppsala University, Signals \& Systems, March 2004.

7. T. Stepinski, "Ultrasonic spectroscopy for the inspection of airspace structures", presented at SPIE's 9th Annual International Symposium on NDE for Health Monitoring and Diagnostics, San Diego, 2-6 March, 2004. 